\title{
Model for a Torsional-Mode Ultrasonic Transducer for an Acousto-Optic In-Fiber Isolator
}

\author{
Gerald T. Moore \\ Air Force Research Laboratory, Directed Energy Directorate, Kirtland Air Force Base Albuquerque, NM 87117, USA \\ Correspondence should be addressed to Gerald T. Moore, gerry.moore@kirtland.af.mil
}

Received 29 October 2009; Accepted 22 January 2010

Academic Editor: Pierre Bourdon

Copyright ( $) 2010$ Gerald T. Moore. This is an open access article distributed under the Creative Commons Attribution License, which permits unrestricted use, distribution, and reproduction in any medium, provided the original work is properly cited.

Torsional ultrasound modes can couple the optical polarization states in a birefringent fiber. Polarization coupling produced by interaction with a higher-order torsional mode slightly above its cutoff may provide a route to producing an in-fiber isolator suitable for use in narrow-band high-power fiber amplifiers. This paper describes a model of a transducer generating torsional modes in a cylindrical fiber. This model predicts that almost all of the power applied to the transducer is radiated into the desired mode. The paper also discusses effects produced by acoustic absorption and the dependence of the acoustic velocity on temperature.

\section{Introduction}

By matching the optical polarization beat length $L_{B}$ in a birefringent silica optical fiber to the axial wavelength of a traveling torsional wave of angular frequency $\Omega$ propagating in the fiber, it is possible to couple light from one polarization to the other $[1,2]$. When light is coupled from the slow polarization to the fast polarization, it is down-shifted in frequency by $\Omega$ if the light and sound are copropagating, but up-shifted if the light and sound are counter-propagating. This nonreciprocal frequency shift can be used to make an infiber acousto-optic ( $\mathrm{AO}$ ) isolator. This requires placing two torsional transducers on the fiber separated by $D=\pi v_{g} / 2 \Omega$, where $v_{g}$ is the group velocity of light in the fast polarization. These transducers propagate sound in the same direction. One watt of acoustic power from each transducer is sufficient to couple $50 \%$ of the light from one polarization to the other over a few centimeters [2]. The polymer jacket is removed from the fiber in the interaction regions to prevent acoustic damping. The relative acoustic phase of the two transducers is adjusted such that in the forward direction all of the light at the wavelength of interest returns to the slow polarization, whereas in the backward direction all of the light at this wavelength goes into the fast polarization. Polarizers at both ends, such as 45-degree tilted fiber Bragg gratings [3], remove light in the fast polarization to make an isolator. To date an isolator based on torsional acoustic waves has not been made, but $100 \%$ polarization switching has been demonstrated using the lowest-order $(s=0)$ torsional mode [1]. However, an analogous isolator was demonstrated using the lowestorder flexural mode at $\Omega / 2 \pi=4.31 \mathrm{MHz}$ [4]. In this case coupling was between the two transverse modes of a twomode fiber. Although a $22 \mathrm{~dB}$ extinction ratio was reported, the large $D=11 \mathrm{~m}$ spacing between the transducers makes this approach unattractive for high-power operation. The key to decreasing this spacing is to increase $\Omega$, while using an acoustic mode for which phase matching is possible at high frequency. Raising the frequency to about $50 \mathrm{MHz}$ reduces the spacing $D$ between transducers to about $1 \mathrm{~m}$. Except for the short regions following the transducers where acoustic coupling occurs, the fiber between the transducers can be coiled and jacketed with polymer. Figure 1 shows the layout of an $\mathrm{AO}$ isolator at four different length scales.

The phase-matching acceptance for polarization switching, which occurs over a few centimeters, is much wider than the transmission features produced by the transducer spacing $D$ and the linear polarizers. The transmission in the forward direction as a function of wavelength $\lambda$ is a comb with periodicity $\lambda L_{B} / D$, while the backward transmission is an out-of-phase comb. This is illustrated in Figure 2. 


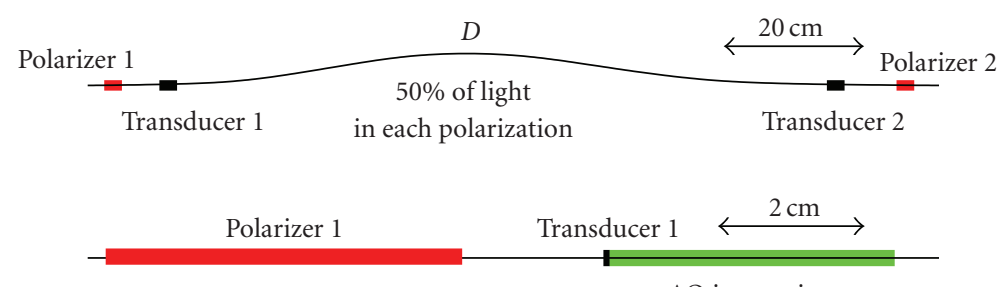

AO interaction

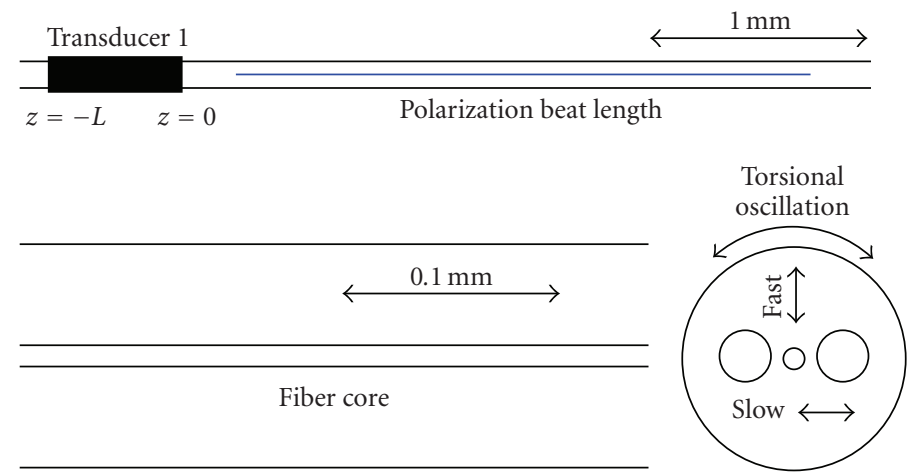

FIGURE 1: Components of an AO isolator are shown schematically at four different length scales. Sizes are approximate. Forward propagating light enters from the left with slow polarization. Polarizers (possibly $45^{\circ}$ tilted fiber Bragg gratings) are oriented to pass the slow polarization. Copropagating torsional acoustic waves generated by transducers act as 50\% polarization couplers. Transducer 2 returns forward light to the slow polarization, while transducer 1 shifts backward light entirely to the fast polarization.

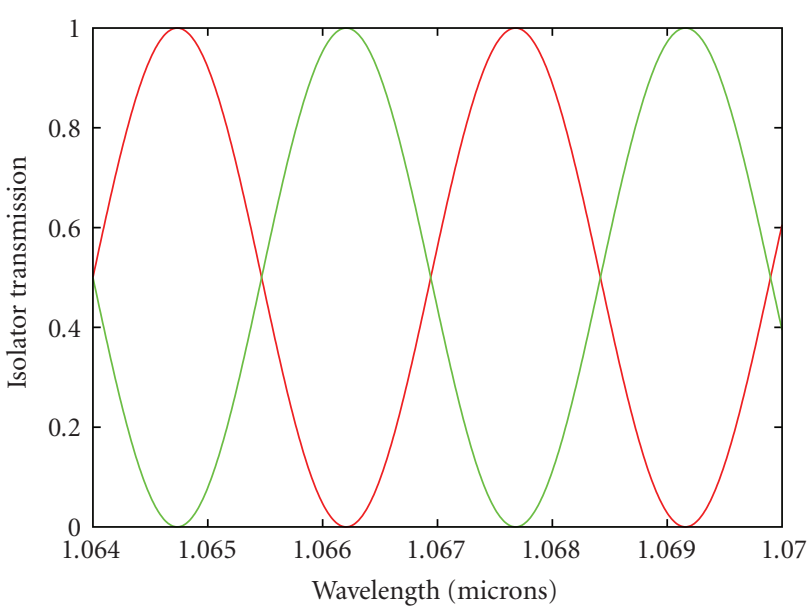

FIGURE 2: Calculated forward (red) and backward (green) transmission through an isolator using fiber with beat length $L_{B}=3 \mathrm{~mm}$ and transducer separation $D=105 \mathrm{~cm}$.

The position of these combs depends on the relative acoustic phase of the two transducers. Hwang et al. [4] found that it was necessary to use a feedback circuit to adjust this phase to compensate for shifting of the combs produced by vibrations or temperature changes. The width of the comb maxima and minima is sufficient to prevent backward propagation of light produced by stimulated Brillouin scattering in narrowband $\mathrm{Yb}$-doped fiber amplifiers. The isolator can be used for multiple wavelengths commensurate with the comb period, such asthose that might be produced by spectral beam combination. However, it cannot protect against inadvertent lasing at other wavelengths within the gain bandwidth of $\mathrm{Yb}$. This type of isolator can be tuned for use with other types of fiber amplifier besides $\mathrm{Yb}$, such as Er or Tm.

A transducer spacing of $1 \mathrm{~m}$ imposes a power limitation on operation of an $\mathrm{AO}$ isolator, since undesirable nonlinear effects can build up over this distance. For example, we estimate that the nonlinear refractive index of fused silica will limit isolator operation to about $200 \mathrm{~W}$ in a $10 \mu \mathrm{m}$ core fiber and about $1 \mathrm{~kW}$ in a $25 \mu \mathrm{m}$ core fiber. By contrast, present fiber-coupled isolators are limited to about $50 \mathrm{~W}$. Bulk isolators can withstand higher power but distort the beam quality at powers above about $100 \mathrm{~W}$.

The frequency for phase-matching of the lowest-order torsional mode is too low for use as a high-power isolator, since $D$ becomes very large and the comb spacing very narrow. However, a transducer producing $100 \%$ polarization coupling with this mode can be used as a tunable notch filter [1], when placed between crossed polarizers. This can filter out undesired wavelengths, including wavelengths within the gain bandwidth of the amplifying medium.

\section{Matching of Transducer Modes and Free-Oscillation Modes}

In the analysis below we consider the fiber to be a homogeneous elastic cylindrical glass rod, neglecting perturbations which might arise because of different elastic properties of the core or the stress rods. Torsional waves in a cylindrical rod are characterized by a displacement $u(r, z)$ which is entirely in the azimuthal direction, but independent of the azimuthal 
coordinate $\theta$. If the cylinder radius is $a$, we can introduce a dimensionless frequency $\gamma=\Omega a / V$, where $V$ is the transverse sound velocity. This velocity is variously reported by Engan to be $3750 \mathrm{~m} / \mathrm{s}$ [2] or $3764 \mathrm{~m} / \mathrm{s}$ [5] in fused silica. Vacher et al. [6] found that $V$ at $5 \mathrm{MHz}$ and $16 \mathrm{GHz}$ are the same to within $0.1 \%$ at room temperature, suggesting that dispersion is small. However, $V$ was found to increase with temperature at $0.3 \mathrm{~m} / \mathrm{s}$ kelvin. It is convenient to express $r$ and $z$ in units of $a$. Then the torsional waves are of the form $u=(1 / 2) r \exp ( \pm i \gamma z)$ or $u=\mathrm{J}_{1}(\alpha r) \exp ( \pm i \beta z)$, where $\beta^{2}=\gamma^{2}-\alpha^{2}$.

The torsional modes of a free elastic cylinder are a subset of all the possible vibrational modes [7]. In general the displacement $u$ has an azimuthal dependence $\cos (n \theta)$ or $\sin (n \theta)$. The modes with $n=0$ can be further subdivided into torsional and axial-radial. The modes with $n=1$ or higher are called flexural. The dispersion relations for the modes, except for the torsional modes, depend on the Poisson ratio for the material. Figure 3 shows the dispersion relations for $n=0$ (red) and $n=1$ (green) for the Poisson ratio 0.17 of fused silica. The lowest and highest red curves shown are the two lowest torsional modes. The other red curves are axial-radial. The lowest torsional, radial-axial, and flexural modes are the only ones with no cutoff. For torsional oscillations the two nonzero components of strain (with proportional components of stress) are $2 \varepsilon_{r \theta}=r \partial(u / r) \partial r$ and $2 \varepsilon_{z \theta}=\partial u / \partial z$ [8]. Our model for the transducer is a section of fiber between $z=-L$ and $z=0$ where $u$ at $r=1$ is a singlefrequency oscillation independent of $z$. The model does not specify how such oscillation is produced. However, at the end of the paper we cite some methods that have been used to excite torsional vibrations in fibers and rods. $\varepsilon_{z \theta}=0$ at the surface of the fiber in the transducer, whereas $\varepsilon_{r \theta}=0$ at the surface of the free fiber. The displacement in the transducer is of the form

$$
\begin{aligned}
u= & \mathrm{J}_{1}(\gamma r) \\
& +\sum_{n=1} \mathrm{~J}_{1}\left(Y_{n} r\right)\left[D_{n} \exp \left(i \kappa_{n}(z+L)\right)+H_{n} \exp \left(-i \kappa_{n} z\right)\right],
\end{aligned}
$$

where $Y_{n}$ is the $n^{\prime}$ th zero of $\mathrm{J}_{1}, \kappa_{n}^{2}=\gamma^{2}-Y_{n}^{2}$, and $\kappa_{n}$ is positive real for propagating modes or positive imaginary for cutoff modes. $D_{n}$ and $H_{n}$ are the modal amplitudes. We could multiply the first term above by a constant, but we choose to normalize in this way for now. Outside the transducer the fiber is uncontrained at the surface; so $\varepsilon_{r \theta}$ should vanish at $r=1$. Since $((r d / d r))\left[(1 / r) \mathrm{J}_{1}(\alpha r)\right]=-\alpha \mathrm{J}_{2}(\alpha r)$, we choose $\alpha=Z_{n}$, where $Z_{n}$ is the $n^{\prime}$ th zero of $\mathrm{J}_{2}$, and write the displacement for $z>0$ in the form

$$
u=\frac{1}{2} C_{0} r \exp (i \gamma z)+\sum_{n=1} C_{n} \mathrm{~J}_{1}\left(Z_{n} r\right) \exp \left(i \beta_{n} z\right),
$$

where $\beta_{n}^{2}=\gamma^{2}-Z_{n}^{2}$ and $\beta_{n}$ is positive real for propagating modes or positive imaginary for cutoff modes. For positive $z$ the propagating modes with modal amplitudes $C_{n}$ carry power only in the positive $z$ direction. The mode with a cutoff at $\gamma=Z_{n}$ we call the $s=n$ mode. Since $Z_{1}=5.136$, the

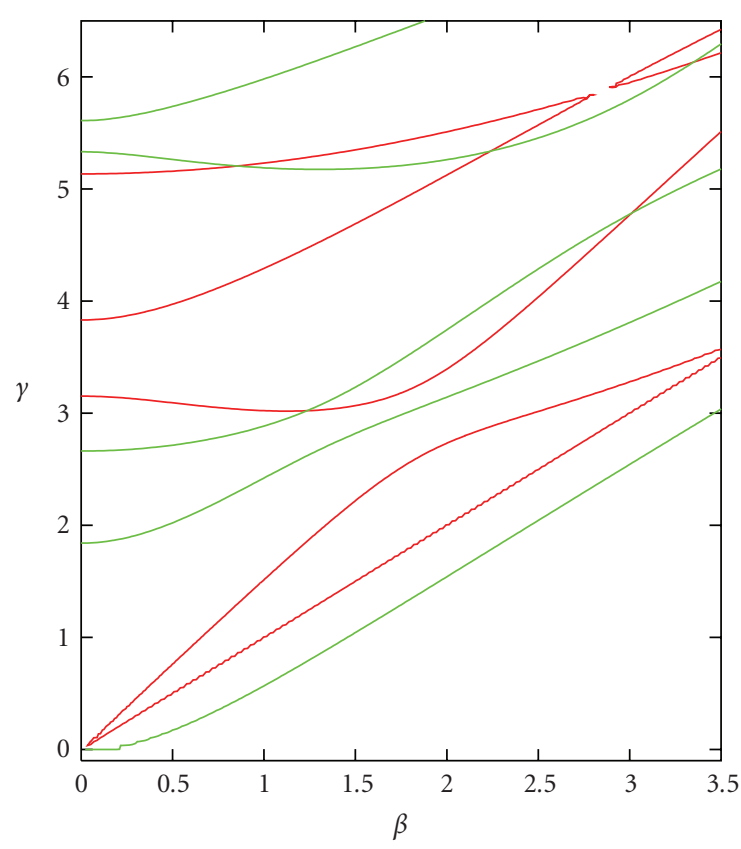

FIGURE 3: Dispersion relations for torsional, radial-axial, and flexural modes for an elastic cylinder. Lowest and highest red curves are torsional; other red curves are axial-radial; green curves are flexural. $\beta$ is longitudinal wave vector in units of $1 / a . \gamma$ is angular frequency in units of $V / a$.

$s=1$ cutoff frequency for a fiber of diameter $2 a=125 \mu \mathrm{m}$ is $\Omega / 2 \pi=V Z_{1} / 2 \pi a=49 \mathrm{MHz}$. By operating just above the cutoff for the $s=n$ mode, we can match the axial wavelength $2 \pi a / \beta_{n}$ to the optical polarization beat length $L_{B}$ (typically about $3 \mathrm{~mm}$ ). The phase velocity $V \gamma / \beta_{n}$ approaches infinity at cutoff, while the sound group velocity $V \beta_{n} / \gamma$ approaches zero. The $s=0$ mode, corresponding to the $C_{0}$ term, has no cutoff, propagates at speed $V$ without dispersion, and can only be phase matched at much lower frequency. Since $Z_{1}$ is greater than $Y_{1}=3.832$, a propagating mode inside the transducer already exists when $\gamma$ passes through the $s=1$ cutoff.

At $z<-L$ we write the displacement as

$$
\begin{aligned}
u= & \frac{1}{2} F_{0} r \exp (-i \gamma(z+L))+\sum_{n=1} F_{n} \mathrm{~J}_{1}\left(Z_{n} r\right) \exp \left(-i \beta_{n}(z+L)\right) \\
& +\frac{1}{2} G_{0} r \exp (i \gamma(z+L))+G_{p} \mathrm{~J}_{1}\left(Z_{p} r\right) \exp \left(i \beta_{p}(z+L)\right) .
\end{aligned}
$$

Here $F_{n}$ are the modal amplitudes for sound propagating in the $-z$ direction. The last two terms with amplitudes $G_{0}$ or $G_{p}$ are inserted to describe reflections of one of the modes above cutoff back into the transducer. For example, if the $s=$ 1 mode is above cutoff, we take $G_{1}$ to be nonzero and $G_{0}=0$. The case of nonzero $G_{0}$ is not of much practical interest but is included for completeness. When the $G$ coefficients are zero, equal acoustic power is radiated from the transducer in 
both directions. The backward power is undesirable. It can be damped by leaving the jacket on the fiber at $z<-L$. However, more efficient operation might be possible by reflecting the power. For a mode near cutoff, reflection can be produced by raising the fiber temperature behind the transducer. This increases $V$, which puts the mode below cutoff in this region. If the reflection is total, then $\left|G_{p}\right|=\left|F_{p}\right|$, but the effects of the reflection depend on the phase $\Theta$ of $G_{p}$. This phase depends on the location of the thermal gradient producing the reflection and cycles through one period when this location shifts by half the longitudinal sound wavelength in the unheated fiber. Under phase-matched conditions this is also half the optical polarization beat length $L_{B}$. Reflection should be produced close to the transducer in order to minimize AO coupling by light going in the $-z$ direction.

At $z=-L$ and $z=0$ both $u$ and $\partial u / \partial z$ should be continuous. This leads to the following set of four equations:

$$
\begin{aligned}
& \mathrm{J}_{1}(\gamma r)+\sum_{n=1}\left(A_{n}-i T_{n} B_{n}\right) \mathrm{J}_{1}\left(Y_{n} r\right) \\
& \quad=\frac{1}{2} F_{0} r+\sum_{n=1} F_{n} \mathrm{~J}_{1}\left(Z_{n} r\right)+\frac{1}{2} G_{0} r+G_{p} \mathrm{~J}_{1}\left(Z_{p} r\right), \\
& \sum_{n=1} \kappa_{n}\left(T_{n} A_{n}+i B_{n}\right) \mathrm{J}_{1}\left(Y_{n} r\right) \\
& \quad=-i \gamma \frac{1}{2} F_{0} r-i \sum_{n=1} \beta_{n} F_{n} \mathrm{~J}_{1}\left(Z_{n} r\right)+i \gamma \frac{1}{2} G_{0} r+i \beta_{p} G_{p} \mathrm{~J}_{1}\left(Z_{p} r\right),
\end{aligned}
$$

$$
\begin{aligned}
& \mathrm{J}_{1}(\gamma r)+\sum_{n=1}\left(A_{n}+i T_{n} B_{n}\right) \mathrm{J}_{1}\left(Y_{n} r\right) \\
& =\frac{1}{2} C_{0} r+\sum_{n=1} C_{n} \mathrm{~J}_{1}\left(Z_{n} r\right), \\
& \sum_{n=1} \kappa_{n}\left(T_{n} A_{n}-i B_{n}\right) \mathrm{J}_{1}\left(Y_{n} r\right) \\
& =-i \gamma \frac{1}{2} C_{0} r-i \sum_{n=1} \beta_{n} C_{n} \mathrm{~J}_{1}\left(Z_{n} r\right) .
\end{aligned}
$$

Here we have introduced

$$
\begin{aligned}
& A_{n}=\frac{1}{2}\left(1+e^{i \kappa_{n} L}\right)\left(D_{n}+H_{n}\right), \\
& B_{n}=\frac{1}{2}\left(1+e^{i \kappa_{n} L}\right)\left(D_{n}-H_{n}\right), \\
& T_{n}=\tan \left(\frac{\kappa_{n} L}{2}\right) .
\end{aligned}
$$

Note that in the absence of reflection $\left(G_{0}=0, G_{p}=0\right)$ we can take $B_{n}=0, F_{0}=C_{0}$, and $F_{n}=C_{n}$, so that emission from the transducer is symmetrical.
The torsional modes outside the transducer are orthogonal, as can be seen from the following identities:

$$
\begin{aligned}
\left(\alpha^{2}-\varepsilon^{2}\right) \int_{0}^{1} d r r \mathrm{~J}_{1}(\alpha r) \mathrm{J}_{1}(\varepsilon r) & =\alpha \mathrm{J}_{1}(\varepsilon) \mathrm{J}_{2}(\alpha)-\varepsilon \mathrm{J}_{1}(\alpha) \mathrm{J}_{2}(\varepsilon) \\
\int_{0}^{1} d r r \mathrm{~J}_{1}^{2}\left(Z_{n} r\right) & =\frac{1}{2} \mathrm{~J}_{1}^{2}\left(Z_{n}\right) \\
\int_{0}^{1} d r r^{2} \mathrm{~J}_{1}(\alpha r) & =\frac{1}{\alpha} \mathrm{J}_{2}(\alpha)
\end{aligned}
$$

Multiplying (4)-(7) by either $r$ or $\mathrm{J}_{1}\left(Z_{m} r\right)$, integrating, and using the above identities, we obtain

$$
\begin{aligned}
& \frac{1}{8}\left(F_{0}+G_{0}\right)=\frac{1}{\gamma} \mathrm{J}_{2}(\gamma)+\sum_{n=1}\left(A_{n}-i T_{n} B_{n}\right) \frac{1}{Y_{n}} \mathrm{~J}_{2}\left(Y_{n}\right), \\
& -\frac{1}{8} i \gamma\left(F_{0}-G_{0}\right)=\sum_{n=1} \kappa_{n}\left(T_{n} A_{n}+i B_{n}\right) \frac{1}{Y_{n}} \mathrm{~J}_{2}\left(Y_{n}\right), \\
& \frac{1}{8} C_{0}=\frac{1}{\gamma} \mathrm{J}_{2}(\gamma)+\sum_{n=1}\left(A_{n}+i T_{n} B_{n}\right) \frac{1}{Y_{n}} \mathrm{~J}_{2}\left(Y_{n}\right), \\
& -\frac{1}{8} i \gamma C_{0}=\sum_{n=1} \kappa_{n}\left(T_{n} A_{n}-i B_{n}\right) \frac{1}{Y_{n}} \mathrm{~J}_{2}\left(Y_{n}\right), \\
& \frac{1}{2} F_{m} \mathrm{~J}_{1}\left(Z_{m}\right)+\frac{1}{2} \delta_{m p} \mathrm{~J}_{1}\left(Z_{p}\right) G_{p} \\
& =\frac{\gamma}{\gamma^{2}-Z_{m}^{2}} \mathrm{~J}_{2}(\gamma)+\sum_{n=1}\left(A_{n}-i T_{n} B_{n}\right) \frac{Y_{n}}{Y_{n}^{2}-Z_{m}^{2}} \mathrm{~J}_{2}\left(Y_{n}\right), \\
& -\frac{1}{2} i \beta_{m} F_{m} \mathrm{~J}_{1}\left(Z_{m}\right)+\frac{1}{2} i \delta_{m p} \beta_{p} \mathrm{~J}_{1}\left(Z_{p}\right) G_{p} \\
& =\sum_{n=1} \kappa_{n}\left(T_{n} A_{n}+i B_{n}\right) \frac{Y_{n}}{Y_{n}^{2}-Z_{m}^{2}} \mathrm{~J}_{2}\left(Y_{n}\right),
\end{aligned}
$$

$\frac{1}{2} C_{m} \mathrm{~J}_{1}\left(Z_{m}\right)$

$$
\begin{array}{r}
=\frac{\gamma}{\gamma^{2}-Z_{m}^{2}} \mathrm{~J}_{2}(\gamma)+\sum_{n=1}\left(A_{n}+i T_{n} B_{n}\right) \frac{Y_{n}}{Y_{n}^{2}-Z_{m}^{2}} \mathrm{~J}_{2}\left(Y_{n}\right), \\
-\frac{1}{2} i \beta_{m} C_{m} \mathrm{~J}_{1}\left(Z_{m}\right)=\sum_{n=1} \kappa_{n}\left(T_{n} A_{n}-i B_{n}\right) \frac{Y_{n}}{Y_{n}^{2}-Z_{m}^{2}} \mathrm{~J}_{2}\left(Y_{n}\right) .
\end{array}
$$

We can eliminate $F_{0}, F_{m}, C_{0}$, and $C_{m}$ from these equations to obtain a set of equations to be obeyed by $A_{n}$ and another set of equations to be obeyed by $B_{n}$. In fact, if we define $Z_{0}=0$ and $\beta_{0}=\gamma$, the equations derived from (12)-(15) can be 
treated on the same footing as those derived from (16)-(19). We obtain

$$
\begin{aligned}
& \frac{1}{8} G_{0}+\frac{1}{2} \delta_{m p} \mathrm{~J}_{1}\left(Z_{p}\right) G_{p} \\
& =\sum_{n=1} B_{n}\left(-i T_{n}+\frac{\kappa_{n}}{\beta_{m}}\right) \frac{Y_{n}}{Y_{n}^{2}-Z_{m}^{2}} \mathrm{~J}_{2}\left(Y_{n}\right), \\
& -\gamma \mathrm{J}_{2}(\gamma)+\frac{1}{8} \gamma^{2} G_{0}+\frac{1}{2} \delta_{m p} \beta_{p}^{2} \mathrm{~J}_{1}\left(Z_{p}\right) G_{p} \\
& =\sum_{n=1} A_{n}\left(1-i \frac{\kappa_{n} T_{n}}{\beta_{m}}\right) \frac{\gamma^{2}-Z_{m}^{2}}{Y_{n}^{2}-Z_{m}^{2}} Y_{n} \mathrm{~J}_{2}\left(Y_{n}\right) .
\end{aligned}
$$

If one first specifies a value of $G_{p}$ (or $G_{0}$ ), these equations constitute a set of linear inhomogeneous equations for the amplitudes $A_{n}$ and $B_{n}$. In order to solve these equations we choose an integer $N$ (typically 200) and truncate by letting $n$ run from 1 to $N$ and $m$ run from 0 to $N-1$. Then we obtain $A_{n}$ and $B_{n}$ by numerical matrix inversion. The coefficients $F_{0}$, $F_{m}, C_{0}$, and $C_{m}$ needed for the modal expansions of $u$ outside the transducer are then obtained from (12), (14), (16), and (18).

If, for example, one is interested in the situation where the $p$ mode is totally reflected at $z<-L$, we repeat the calculation for different values of $\left|G_{p}\right|$ in order to home in on the value of $\left|G_{p}\right|$ for which $\left|G_{p}\right|=\left|F_{p}\right|$. The result depends on the phase $\Theta$ of $G_{p}$, which in turn depends on how and where the reflection occurs.

To check the convergence of our numerical procedure, we can plot the real and imaginary parts of the left and right sides of (4)-(7) as a function of $r$ to see if they match. Equations (4) and (6) represent the displacements $u(-L, r)$ and $u(0, r)$, while (5) and (7) are proportional to the strain $\varepsilon_{z \theta}$. We can also calculate $-r \partial(u / r) \partial r$ from (4) and (6) to obtain expressions proportional to the strain $\varepsilon_{r \theta}$. The real parts correspond to times when the transducer amplitude (the displacement at $r=1$ ) is maximal, while the imaginary parts correspond to times when the transducer amplitude is zero. In making these plots the number of terms we retain in the sums (typically 140) is less than $N$, since the final terms can be corrupted by the matrix truncation.

We find that the matching of the external and internal displacements and strains is good. The differences appear as low-amplitude, high-frequency oscillations. The differences are most apparent in $\varepsilon_{z \theta}$, which is zero at $r=1$ in the transducer but appears to be discontinuous as a function of $z$ at $z=-L$ and $z=0$ when $r=1$. The displacements and strains are insensitive to the choice of $N$ if $N$ is not too small.

\section{Energy Considerations}

Since our model neglects sound absorption in the fiber, the power radiated in the modes which are above cutoff should equal the power $P_{T}$ applied to the transducer. Modes below cutoff are exponentially attenuated away from the transducer and do not propagate power. Let $U$ be the amplitude of the transducer motion at $r=1$. Then the modal coefficients calculated in the previous section should be multiplied by
$U /\left|J_{1}(\gamma)\right|$ to determine the physical displacement amplitudes. By comparison with the power expressions given in [2], we see that the power propagating in the $s=0$ mode at $z>0$ is $P_{s=0,+}=\left[\gamma\left|C_{0}\right| / 2 \mathrm{~J}_{1}(\gamma)\right]^{2} \mathcal{P}$, where $\mathcal{P}=(1 / 4) \pi \rho V^{3} U^{2}$ and $\rho=2200 \mathrm{~kg} / \mathrm{m}^{2}$ is the density of fused silica. If, for example, $U=90 \mathrm{~nm}$, then $\mathcal{P}=0.738 \mathrm{~W}$. The power in higher-order modes which are above cutoff is $P_{s=n,+}=$ $2\left[\left|C_{n}\right| \mathrm{J}_{1}\left(Z_{n}\right) / \mathrm{J}_{1}(\gamma)\right]^{2} \beta_{n} \gamma \mathcal{P}$. Analogous expressions apply to the power propagating at $z<-L$.

Division by $\left|J_{1}(\gamma)\right|$ is not possible when $\gamma=Y_{n}$ for some $n$. In this case there is no steady state in the absence of damping. When $Y_{1}<\gamma<Z_{1}$, there is one propagating mode inside the transducer. We call this the $t=1$ mode. This mode can radiate only to the $s=0$ mode outside the transducer, and resonances occur as a function of $L$. Internal values of $u$ are typically large compared to $u$ at the surface, and the $t=1$ power within the transducer can be much larger than the power driving the transducer. When $\gamma$ exceeds $Z_{1}$, the $s=1$ channel opens, sound vibrations are no longer trapped in the transducer, the $t=1$ power is small, and the generated $s=1$ power is almost independent of $L$. In general the $t=n$ power propagating in the forward and backward direction is given for modes above cutoff by

$$
P_{t=n, \pm}=\frac{1}{2} \mathcal{P} \gamma \kappa_{n}\left[\frac{\mathrm{J}_{2}\left(Y_{n}\right)\left|A_{n} \pm B_{n}\right|}{\mathrm{J}_{1}(\gamma) \cos \left(\kappa_{n} L / 2\right)}\right]^{2} .
$$

To calculate the time-averaged power driving the transducer we use the stress

$$
\begin{aligned}
\sigma_{r \theta}= & 2 \rho V^{2} \varepsilon_{r \theta} \\
= & -\frac{\rho V^{2} U}{a\left|\mathrm{~J}_{1}(\gamma)\right|}\left\{\gamma \mathrm{J}_{2}(\gamma) \cos (\Omega t)\right\} \\
& +\operatorname{Re}\left\{\sum _ { n = 1 } Y _ { n } \mathrm { J } _ { 2 } ( Y _ { n } ) \left[D_{n} \exp \left(i \kappa_{n}(z+L)\right)\right.\right. \\
& \left.\left.+H_{n} \exp \left(-i \kappa_{n} z\right)\right] \exp (-i \Omega t)\right\}
\end{aligned}
$$

evaluated at the surface of the fiber. The time-averaged power $d P_{T}$ driving the transducer over the surface area $a^{2} d \theta d z$ is obtained by multiplying this stress by the surface velocity $-U \Omega \sin (\Omega t)$ and averaging over time,

$$
\begin{aligned}
d P_{T}= & \frac{1}{2} a d \theta d z \frac{U^{2} \rho V^{2} \Omega}{\left|\mathrm{J}_{1}(\gamma)\right|} \\
& \times \sum_{n=1} Y_{n} \mathrm{~J}_{2}\left(Y_{n}\right) \operatorname{Im}\left[D_{n} \exp \left(i \kappa_{n}(z+L)\right)\right.
\end{aligned}
$$

$$
\left.+H_{n} \exp \left(-i \kappa_{n} z\right)\right]
$$

Integrating over the surface of the transducer and using (8) and (10) yields

$$
P_{T}=8 \mathcal{P} \frac{\gamma}{\left|\mathrm{J}_{1}(\gamma)\right|} \sum_{n=1} Y_{n} \mathrm{~J}_{2}\left(Y_{n}\right) \operatorname{Im}\left(\frac{T_{n} A_{n}}{\kappa_{n}}\right) .
$$

It is often more meaningful to express powers relative to $P_{T}$ than in terms of $\mathcal{P}$. 


\section{Numerical Results}

Let us begin by considering a transducer with $L=10$ and $\gamma=5.13767$, slightly above the $s=1$ cutoff. For a fiber with a diameter of $2 a=125 \mu \mathrm{m}$ this corresponds to a frequency $\Omega / 2 \pi=49.06 \mathrm{MHz}$ and an axial $s=1$ wavelength $2 \pi a / \beta_{1}=$ $2.7 \mathrm{~mm}$, on the order of the polarization beat length $L_{B}$ in polarization-maintaining optical fibers. One watt of power in this mode corresponds to an amplitude at $r=1$ of $85.7 \mathrm{~nm}$. At this frequency the $s=0$ wavelength $2 \pi a / \gamma=76.4 \mu \mathrm{m}$ is much too small for phase matching. We wish to compare the situation where the sound is emitted equally in both directions to the case where the $s=1$ mode is perfectly reflected at $z=-L$ with phase $\Theta=0$ or $180^{\circ}$. $U$ is held constant in Figures 4 and 5 , but $P_{T}$ depends on whether there is reflection. The red curves are at $z=0$ with reflection, the green curves are at $z=-L$ with reflection, and the blue curves are at either end with no reflection.

In the case $\Theta=0, P_{T}=1.47 \mathcal{P}$ with reflection, but $P_{T}=$ $2.96 \mathcal{P}$ with no reflection. Thus, reflection acts to reduce the power needed to drive the transducer to a given amplitude $U$ but does not greatly affect the way the transducer vibrates. The modal efficiencies with reflection are $P_{s=0,+} / P_{T}=0.56 \%$, $P_{s=1,+} / P_{T}=98.64 \%$, and $P_{s=0,-} / P_{T}=0.07 \% . P_{s=1,-} / P_{T}=$ $25.76 \%$ propagates in each direction between the point of reflection and $z=-L$. Within the transducer $P_{t=1,+} / P_{T}=$ $0.028 \%$ and $P_{t=1,-} / P_{T}=0.776 \%$. The amplitude at $r=1$ of the $s=1$ mode is $0.985 U$.

In the case $\Theta=180^{\circ}, P_{T}=120.60 \mathcal{P}$; so it becomes much harder to drive the transducer to a given amplitude $U$. The modal efficiencies are $P_{s=0,+} / P_{T}=58.76 \%, P_{s=1,+} / P_{T}=$ $4.94 \%$, and $P_{s=0,-} / P_{T}=36.18 \%$. Thus, the transducer becomes a poor emitter of $s=1$ ultrasound, although a large power $P_{s=1,-} / P_{T}=1943.6 \%$ propagates in each direction in the region $z<-L$ between the point of reflection and the left end of the transducer. Within the transducer $P_{t=1,+} / P_{T}=$ $63.71 \%$ and $P_{t=1,-} / P_{T}=2.02 \%$. The transducer motion is qualitatively quite different because of the reflection.

In Figure 6 we plot the efficiency $P_{s=1,+} / P_{T}$ as a function of $\Theta$. We see that the efficiency is high over a broad range. On the other hand, without reflection $49.73 \%$ of the applied power $P_{T}$ is emitted into the $s=1$ mode in each direction, and the undesirable backward sound must be damped to prevent interaction with light in the fiber core.

The transducer simulations described below are for no reflections at $z<-L$. Above the $s=1$ cutoff the applied power $P_{T}$ and the radiated power are only weakly dependent on $L$. Figure 7 shows the $s=1$ efficiency for each direction as a function of $L$ at $\gamma=5.13767$. The $s=0$ and internal $t=1$ powers are much smaller than the $s=1$ power. The situation is different below the $s=1$ cutoff, where acoustic energy can leave the transducer only in the $s=0$ channel. This is illustrated in Figure 8 for $\gamma=5.12$, such that $2 \pi / \kappa_{1}=1.85$. While the efficiency for generating the $s=0$ mode in each direction remains at $1 / 2$ (red), $P_{T}$ goes to zero at resonances when $L$ is a multiple of $2 \pi / \kappa_{1}$ (blue). Consequently the ratio $P_{t=1} / P_{T}$ becomes large near these resonances (green). We expect that damping effects not included in our model will act to suppress these resonances, but it may be best to avoid them by a suitable choice of L.

In order to increase the frequency $\Omega$, so as to shorten the distance between the two transducers in an isolator, or to use a larger-diameter fiber, it is of interest to consider increasing $\gamma$ above the cutoff for higher-order modes. Figure 9 shows the $s=2$ efficiency as a function of $L$ at $\gamma=8.41849$, slightly above the $Z_{2}=8.41724$ cutoff (red). This figure also shows the $s=3$ efficiency at $\gamma=11.62075$, slightly above the $Z_{3}=$ 11.61984 cutoff (green). We see that, as desired, most of the acoustic power goes into the mode that is just above cutoff. Scaling to large $\Omega$ will eventually be limited by the increased attenuation of ultrasound at very high frequencies.

At $\gamma=8.416$, just below the $s=2$ cutoff, there is no dominance of $s=1$ over $s=0$. Figure 10 shows $P_{s=0} / P_{T}$ (red) and $P_{s=1} / P_{T}$ (green), as well as the internal resonances $P_{t=1} / P_{T}$ (blue) and $P_{t=2} / P_{T}$ (violet). The $s=0$ and $s=1$ efficiencies in each direction sum to $1 / 2$.

\section{Acousto-Optical Coupling, Acoustic Attenuation, and Thermal Effects}

According to Engan [2] the coupling of the optical polarization states by phase-matched torsional acoustic waves is sinusoidal. That is, one polarization amplitude increases as $\sin \left(K_{n} z\right)$, while the other decreases as $\cos \left(K_{n} z\right)$. Here $z$ is position (not in units of $a$ ) along the fiber. We call $K_{n} z$ the coupling angle. The coupling constant $K_{n}$ for the $s=n$ mode, provided that mode is above cutoff, is

$$
\begin{aligned}
& K_{0}=\left(\frac{24.7}{\mathrm{~m}}\right)\left(\frac{P}{\mathrm{~W}}\right)^{1 / 2}\left[\frac{125 \mu \mathrm{m}}{(2 a)}\right]^{2}, \\
& K_{n}=K_{0} \frac{Z_{n}}{2 \sqrt{2}\left|\mathrm{~J}_{1}\left(Z_{n}\right)\right|}\left[1+\left(\frac{Z_{n}}{\beta_{n}}\right)^{2}\right]^{-1 / 4},
\end{aligned}
$$

where $P$ is the acoustic power injected at $z=0$ into the phase-matched mode. Coupling of $50 \%$ of the light from one polarization to the other occurs over a distance $L_{c}=\pi /\left(4 K_{n}\right)$. For $1 \mathrm{~W}$ of power in a fiber with a $2.7 \mathrm{~mm}$ polarization beat length and a $125 \mu \mathrm{m}$ diameter, the coupling length for the $s=1$ mode is $L_{c}=3.54 \mathrm{~cm}$ and the phase-matching frequency is $49.06 \mathrm{MHz}$. For the same power and beat length, but a $250 \mu \mathrm{m}$ diameter, the coupling length for the $s=4$ mode is $L_{c}=3.58 \mathrm{~cm}$ and the phase-matching frequency is 70.66 MHz.

When one takes damping of the sound waves into account, the power decreases exponentially after the sound leaves the transducer at $z=0$. This means that $\beta_{n}$ has an imaginary part even above cutoff. The coupling angle at position $z$ now becomes $\left[K_{n} a / \operatorname{Im}\left(\beta_{n}\right)\right]\left[1-\exp \left(-\operatorname{Im}\left(\beta_{n}\right) z / a\right)\right]$. This means that a coupling angle of $\pi / 4$ is only possible if $L_{c}<L_{a}$, where the absorption length is defined as $L_{a}=$ $a / \operatorname{Im}\left(\beta_{n}\right)$. The acoustic power decays as $\exp \left(-2 z / L_{a}\right)$. When there is damping the distance over which sufficient coupling occurs is greater than $L_{c}$.

We assume that the damping of the $s=0$ mode is the same as that found for transverse sound waves in bulk fused 


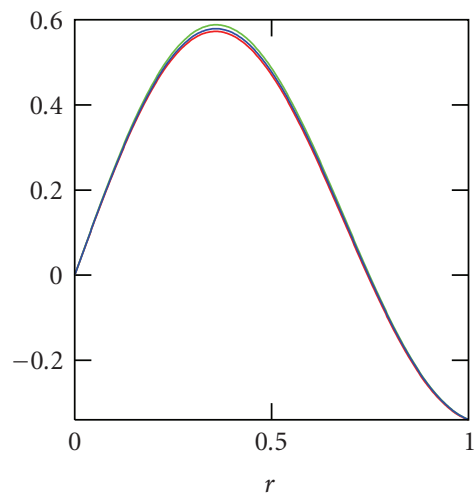

(a)

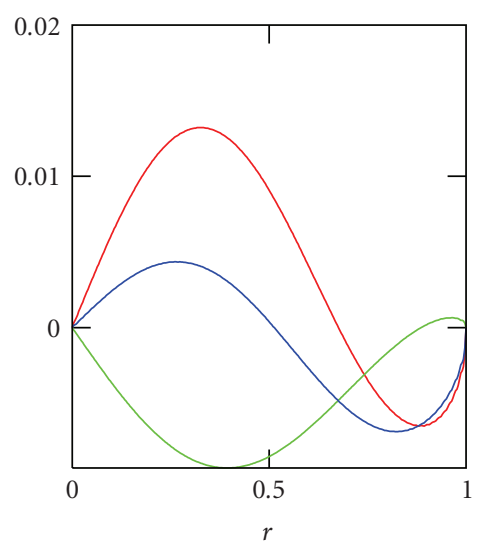

(d)

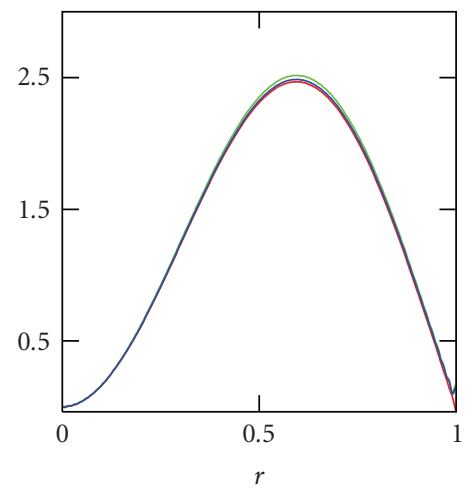

(b)

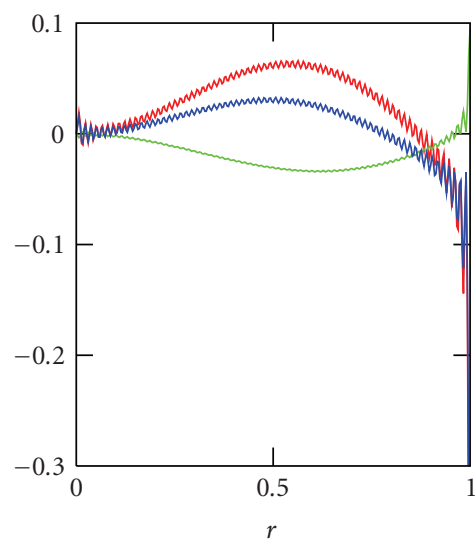

(e)

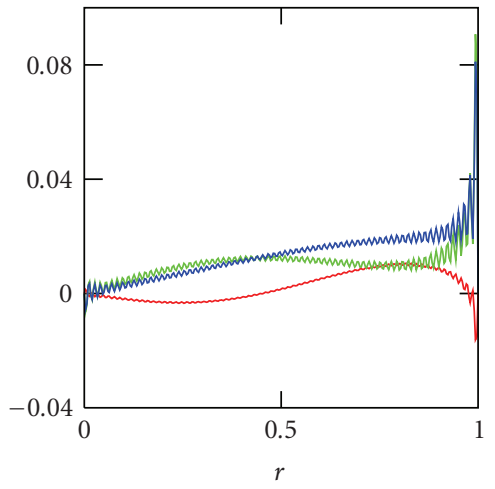

(c)

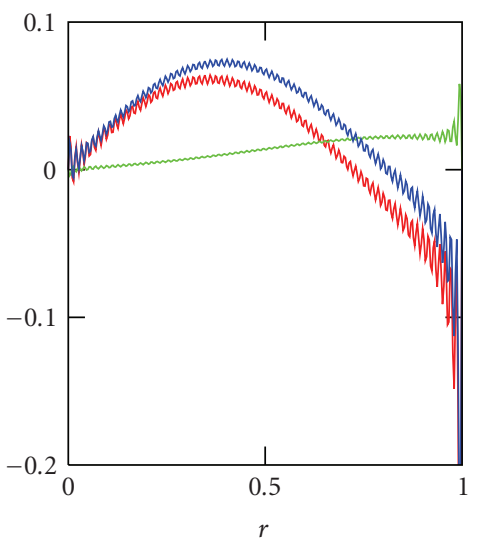

(f)

Figure 4: Displacement $u(r)$ (a) and (d), strain $\varepsilon_{r \theta}(r)(\mathrm{b})$ and (e), and strain $\varepsilon_{z \theta}(r)(\mathrm{c})$ and (f) are shown at the two ends of the transducer. Transverse coordinate $r$ is relative to the fiber radius $a$. Scaling is arbitrary. The normalized frequency is $\gamma=5.13767$. Red $(z=0)$ and green $(z=-L)$ curves are for unit reflectivity of the $s=1$ mode back towards $z=-L$ with phase $\Theta=0$. Blue curve is for zero reflectivity and the same transducer amplitude $U$. (a), (b), (c), and (d) show the real parts, corresponding to times when $u(1)=U$. (d), (e), and (f) show the imaginary parts, corresponding to times when $u(1)=0$. High-frequency oscillations in some of the curves are a numerical artifact. Since $\varepsilon_{z \theta}(r)$ in the absence of reflection has opposite signs at $z=0$ and $z=-L$, the right-hand green plots have been multiplied by minus 1 for better comparison with the blue plots.

silica. Room-temperature measurements by Krischer [9] in the 200 to $1000 \mathrm{MHz}$ range found the power attenuation to be $5.7 \times 10^{-18} \mathrm{~dB} / \mu \sec (f / \mathrm{H} z)^{2}$, where $f=\Omega / 2 \pi$. This agrees well with the value given by Kul'bitskaya and Shutilov [10] but is smaller than the value $1.4 \times 10^{-17} \mathrm{~dB} / \mu \sec (f / \mathrm{Hz})^{2}$ reported by Fraser et al. [11]. Measurements by Krause [12] at $590 \mathrm{MHz}$ showed a decrease in attenuation with increasing temperature with a minimum at about 375 C. For the present discussion we assume that Krischer's result can be extrapolated to lower frequencies. Dividing by $V$ and neglecting dispersion in the speed of sound, we find that $\operatorname{Im}\left(\beta_{0}\right) / a=4.43 \times 10^{-18} \Omega^{2} \mathrm{sec}^{2} / \mathrm{m}$ or $\beta_{0}=\gamma+i\left(a_{0} / a\right) \gamma^{2}$, where $a_{0}=6.63 \times 10^{-11} \mathrm{~m}$.

For the $s=0$ mode $\beta_{0} / a$ is the total wave vector, and the transverse wave vector is zero. For higher-order modes we take $\beta_{n}=\left(\beta_{0}^{2}-Z_{n}^{2}\right)^{1 / 2}$, since the transverse wave vector is $Z_{n} / a$. Note that damping smears out the cutoff, and that $\beta_{n}$ has both real and imaginary parts on either side of the cutoff. Suppose that the beat length is $L_{B}=2.7 \mathrm{~mm}$. For the example above of $2 a=125 \mu \mathrm{m}$ and $f=49.06 \mathrm{MHz}$, the absorption length for the $s=1$ mode is $L_{a}=6.32 \mathrm{~cm}$. The minimum power required at $z=0$ to achieve $50 \%$ optical coupling is $P=0.313 \mathrm{~W}$. The $s=2$ mode in this fiber at $80.39 \mathrm{MHz}$ has $L_{a}=1.44 \mathrm{~cm}$ and requires at least $P=$ $2.36 \mathrm{~W}$. For the example $2 a=250 \mu \mathrm{m}$ and $f=70.66 \mathrm{MHz}$, the absorption length for the $s=4$ mode is $L_{a}=2.12 \mathrm{~cm}$ and at least $2.87 \mathrm{~W}$ in this mode is required. Absorbed acoustic power can produce a temperature gradient along the fiber. This can cause a chirp in $\beta_{n}$, adversely affecting phase matching with the optical field. On the other hand, the phase-matching acceptance increases when the AO coupling length decreases. Heating can also be caused by optical power in the cladding. Cladding light can be removed before the isolator by embedding a section of fiber in high-index material. Air flow past the fiber can also reduce temperature gradients.

Even in the absence of temperature gradients and acoustic damping, the temperature dependence of $V$ [6] affects 


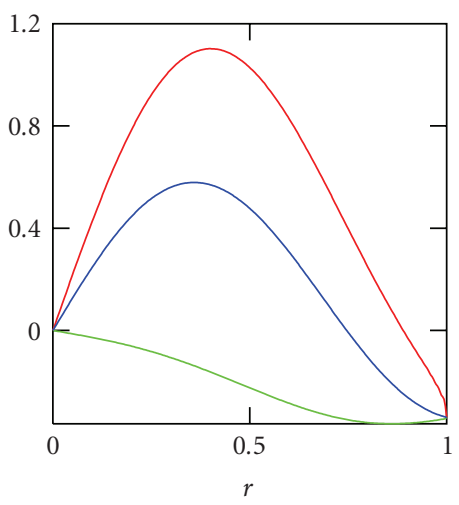

(a)

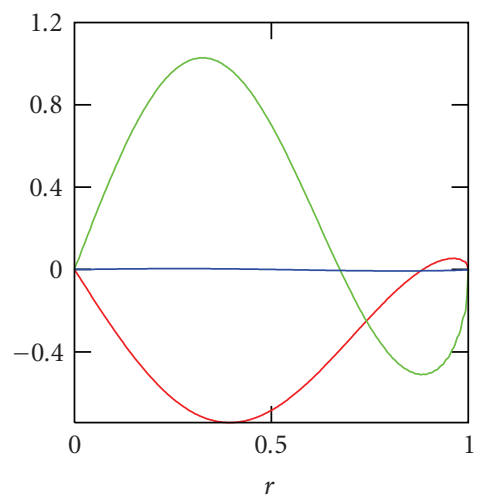

(d)

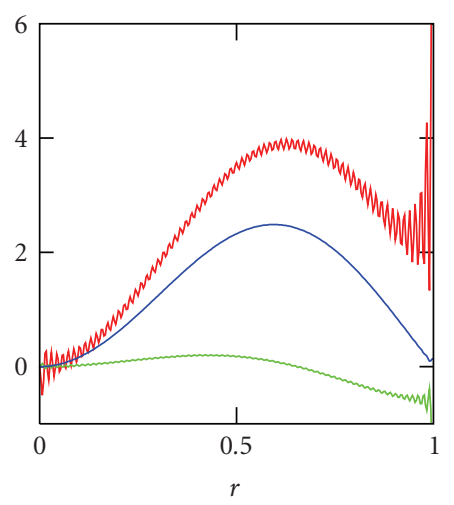

(b)

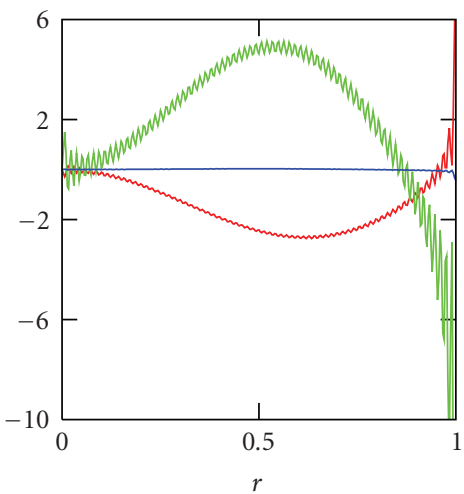

(e)

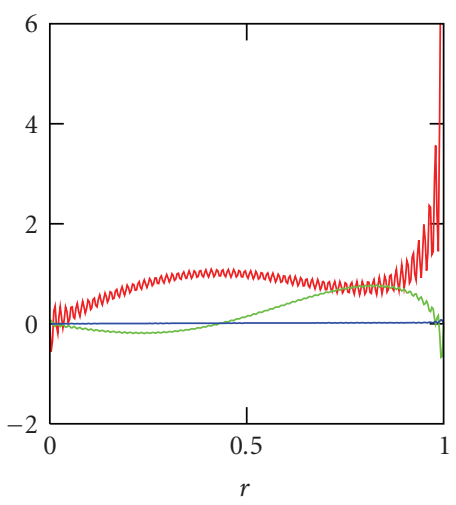

(c)

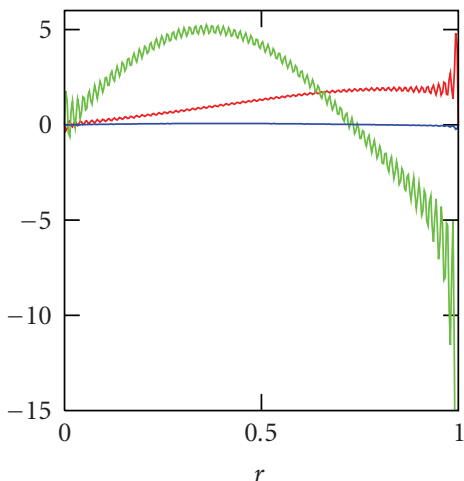

(f)

Figure 5: Displacement $u(r)$ (a) and (d), strain $\varepsilon_{r \theta}(r)(\mathrm{b})$ and (e), and strain $\varepsilon_{z \theta}(r)$ (c) and (f) are shown at the two ends of the transducer. This is for the same conditions as in Figure 4, except that the phase of the reflected $s=1$ mode is $180^{\circ}$.

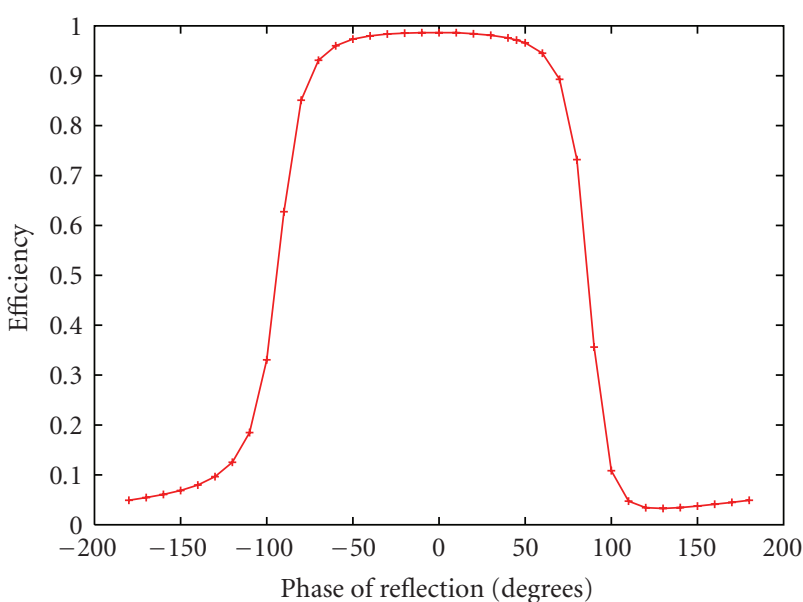

Figure 6: Efficiency $P_{s=1,+} / P_{T}$ as a function of the phase $\Theta$ of the reflected $s=1$ mode at $z=-L$.

the cutoff frequencies and the phase-matching conditions. Figure 11 shows the axial sound wavelength $2 \pi a /\left|\beta_{1}\right|$ as a function of temperature for fibers with diameters 124.9 to $125.2 \mu \mathrm{m}$. The frequency $f=49.2406 \mathrm{MHz}$ is chosen to give an axial wavelength of $3 \mathrm{~mm}$ at $20 \mathrm{C}$ in a $125.0 \mu \mathrm{m}$ fiber. To the right of the cutoff resonances the ordinate is

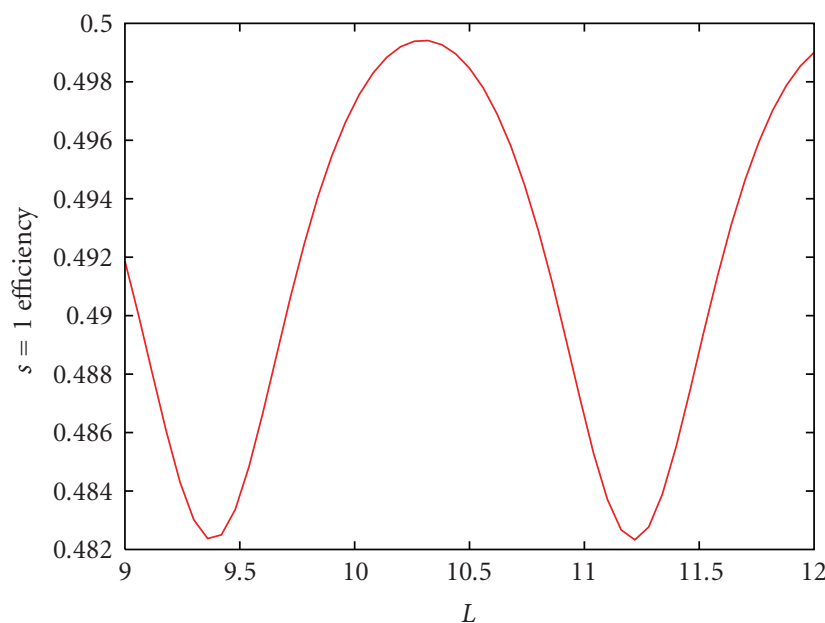

FIGURE 7: Efficiency for $s=1$ generation at $\gamma=5.13767$. Transducer length $L$ is in units of $a$.

the distance over which the power decreases by $1 / e^{2}$. If the fiber diameter were to be slightly different at the locations of the two transducers in an isolator, phase matching for both transducers at the same frequency could be maintained by having the transducers at different temperatures. Backward acoustic power produced by a transducer can be reflected 


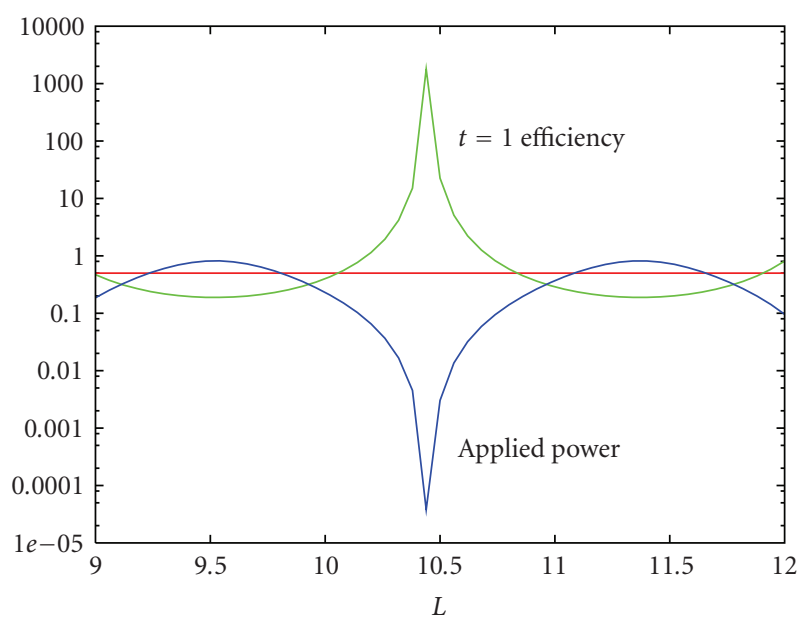

Figure 8: Efficiency for $s=0$ generation (red) at $\gamma=5.12$ remains at $1 / 2$, but $P_{T}$ (blue) becomes small near resonances. As a result the internal $t=1$ efficiency $P_{t=1} / P_{T}$ (green) is large at the resonances.

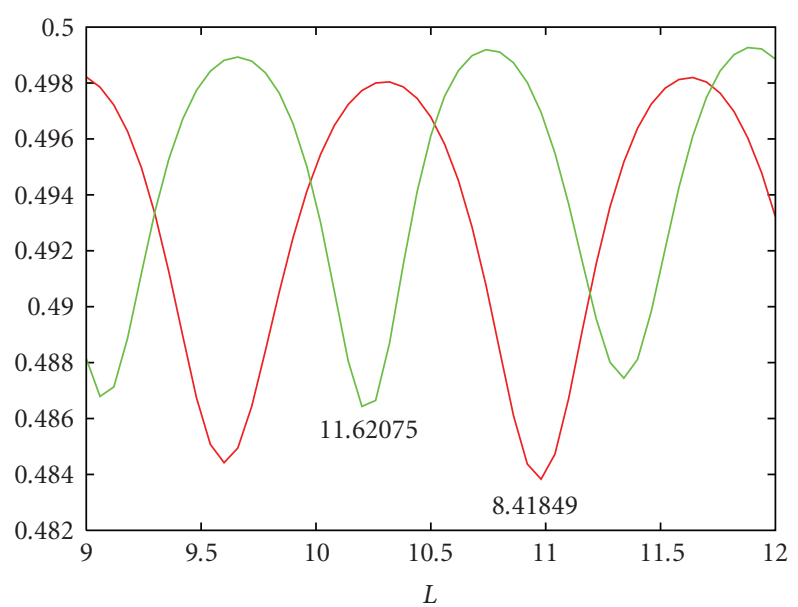

FIGURE 9: Efficiencies for generating the $s=2$ mode (red) and the $s=3$ mode slightly above their cutoffs.

by heating the fiber behind the transducer over a sufficient length to prevent tunneling through the region where the reflected mode is below cutoff. The phase $\Theta$ of the reflected light depends on how and where this heating is done.

\section{Discussion}

Using higher-order torsional acoustic modes near cutoff to couple optical polarization states in a birefringent fiber allows phase matching to the polarization beat length at high frequencies in the $50 \mathrm{MHz}$ range. Two torsionalmode transducers producing $50 \%$ polarization coupling and separated on the fiber by about $1 \mathrm{~m}$ can be used as an in-fiber optical isolator suitable for use with narrow-band fiber amplifiers of arbitrary wavelength. We have presented an analytical and numerical model of a torsional-mode transducer. This model indicates that the mode just above cutoff radiates most of the acoustic power applied to the

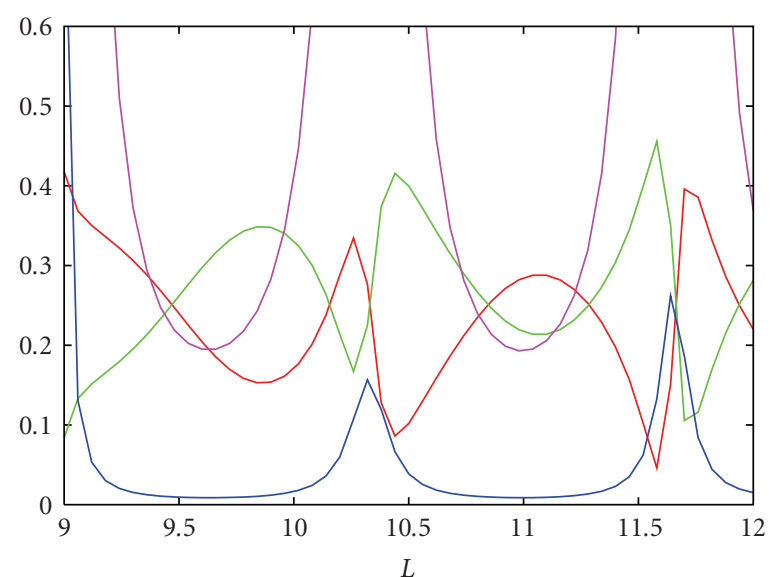

Figure 10: Efficiencies for generating the $s=0$ mode (red), the $s=1$ mode (green), the $t=1$ internal resonance (blue), and the $t=2$ internal resonance (violet) slightly below the $s=2$ cutoff.

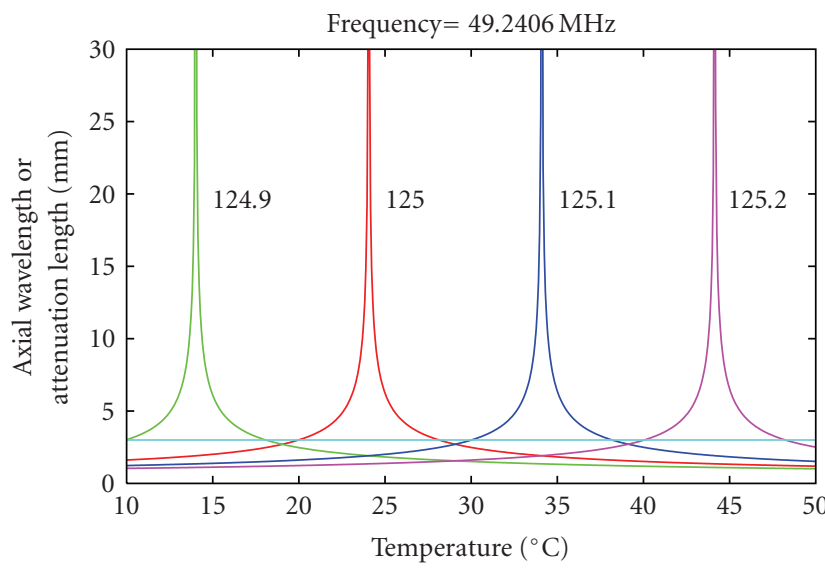

FIGURE 11: Axial wavelength (left of cutoff resonances) and $1 / e^{2}$ attenuation length (right of resonances) is shown as a function of temperature for several fiber diameters. The frequency is chosen to produce an axial wavelength of $3 \mathrm{~mm}$ at $20 \mathrm{C}$ for a $125.0 \mu \mathrm{m}$ diameter fiber.

transducer, irrespective of the transducer length. In the absence of acoustic reflection the power is radiated equally in both directions, and undesirable backward radiation must be absorbed. Reflecting the backward radiation towards the transducer with a suitable phase can enhance the efficiency in the forward direction to almost $100 \%$.

Various types of transducers have been developed for exciting torsional vibrations in fibers or rods, but not at the high frequencies needed for an $\mathrm{AO}$ isolator. Transducers for exciting the $s=0$ mode in optical fibers have used a conical glass horn with the fiber mounted along its axis and shear-mode piezoelectric transducers attached to its base $[1,13-15]$. Other torsional transducers have been developed which use paired [16] or segmented $[17,18]$ piezoelectric elements. Torsional oscillations in metallic rods have been produced by electromagnetic-acoustic transduction [19]. Silicon-based micro-electromechanical systems (MEMSs) technology may provide a route to making higher-frequency 
torsional transducers. We are working with Sandia National Laboratories to explore this possibility.

\section{Acknowledgments}

The author would like to thank Chunte A. Lu for useful discussions. This research was supported in part by the HighEnergy Laser Joint Technology Office.

\section{References}

[1] K. J. Lee, H. C. Park, H. S. Park, and B. Y. Kim, "Highly efficient all-fiber tunable polarization filter using torsional acoustic wave," Optics Express, vol. 15, no. 19, pp. 12362-12367, 2007.

[2] H. E. Engan, "Analysis of polarization-mode coupling by acoustic torsional waves in optical fibers," Journal of the Optical Society of America A, vol. 13, no. 1, pp. 112-118, 1996.

[3] K. Zhou, G. Simpson, X. Chen, L. Zhang, and I. Bennion, "High extinction ratio in-fiber polarizers based on $45^{\circ}$ tilted fiber Bragg gratings," Optics Letters, vol. 30, no. 11, pp. 12851287, 2005.

[4] I. K. Hwang, S. H. Yun, and B. Y. Kim, "All-fiber-optic nonreciprocal modulator," Optics Letters, vol. 22, no. 8, pp. 507-509, 1997.

[5] H. E. Engan, B. Y. Kim, J. N. Blake, and H. J. Shaw, "Propagation and optical interaction of guided acoustic waves in two-mode optical fibers," Journal of Lightwave Technology, vol. 6, no. 3, pp. 428-436, 1988.

[6] R. Vacher, J. Pelous, F. Plicque, and A. Zarembowitch, "Ultrasonic and brillouin scattering study of the elastic properties of vitreous silica between 10 and $300 \mathrm{~K}$," Journal of NonCrystalline Solids, vol. 45, no. 3, pp. 397-410, 1981.

[7] R. N. Thurston, "Elastic waves in rods and clad rods," Journal of the Acoustical Society of America, vol. 64, no. 1, pp. 1-37, 1978.

[8] A. I. Beltzer, Acoustics of Solids, Springer, Berlin, Germany, 1988.

[9] C. Krischer, "Optical measurements of ultrasonic attenuation and reflection losses in fused silica," Journal of the Acoustical Society of America, vol. 48, pp. 1086-1092, 1970.

[10] M. N. Kul'bitskaya and V. A. Shutilov, "Ultrasonic investigation of glasses (review)," Soviet Physics-Acoustics, vol. 22, pp. 451-461, 1976.

[11] D. B. Fraser, J. T. Krause, and A. H. Meitzler, "Physical limitations on the performance of vitreous silica in highfrequency ultrasonic and acousto-optical devices," Applied Physics Letters, vol. 11, no. 10, pp. 308-310, 1967.

[12] J. T. Krause, "Gold-indium bond for measurement of ultrasonic properties in solids at high temperatures," Journal of Applied Physics, vol. 39, no. 11, pp. 5334-5335, 1968.

[13] K. J. Lee, K. S. Hong, H. C. Park, and B. Y. Kim, "Polarization coupling in a highly birefringent photonic crystal fiber by torsional acoustic wave," Optics Express, vol. 16, no. 7, pp. 4631-4638, 2008.

[14] M. Berwick, C. N. Pannell, P. ST. J. Russell, and D. A. Jackson, "Demonstration of birefringent optical fibre frequency shifter employing torsional acoustic waves," Electronics Letters, vol. 27, no. 9, pp. 713-715, 1991.

[15] M. Berwick and D. A. Jackson, "Coaxial optical-fiber frequency shifter," Optics Letters, vol. 17, pp. 270-272, 1992.

[16] J. Friend, K. Nakamura, and S. Ueha, "A torsional transducer through in-plane shearing of paired planar piezoelectric elements," IEEE Transactions on Ultrasonics, Ferroelectrics, and Frequency Control, vol. 51, no. 7, pp. 871-878, 2004.

[17] N. Tsubouchi, "Resonator for torsional vibration," US patent no. 3,774,057, November 1973.

[18] J. O. Kim and O. S. Kwon, "Vibration characteristics of piezoelectric torsional transducers," Journal of Sound and Vibration, vol. 264, no. 2, pp. 453-473, 2003.

[19] W. Johnson, B. A. Auld, and G. A. Alers, "Spectroscopy of resonant torsional modes in cylindrical rods using electromagnetic-acoustic transduction," Journal of the Acoustical Society of America, vol. 95, no. 3, pp. 1413-1418, 1994. 

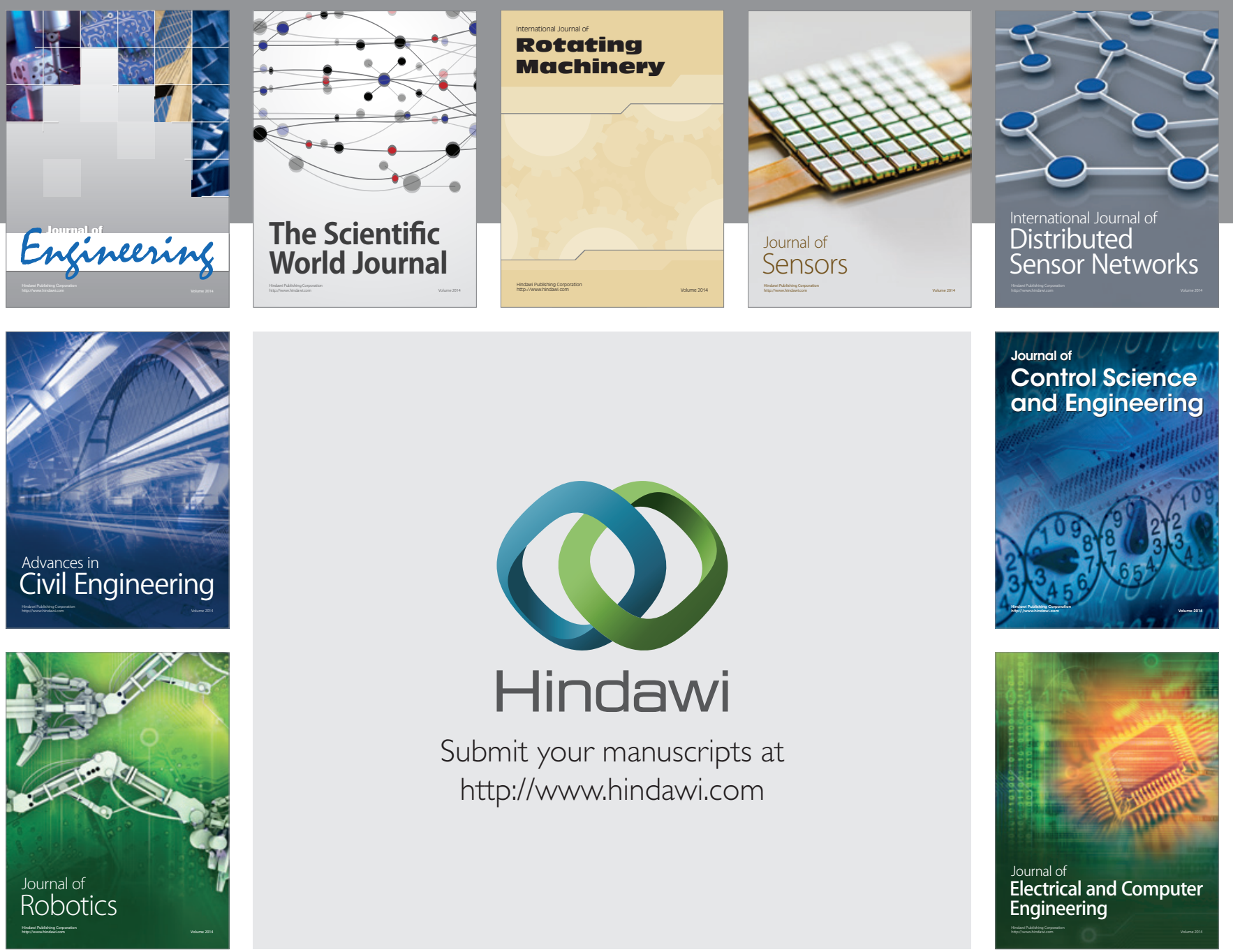

Submit your manuscripts at

http://www.hindawi.com
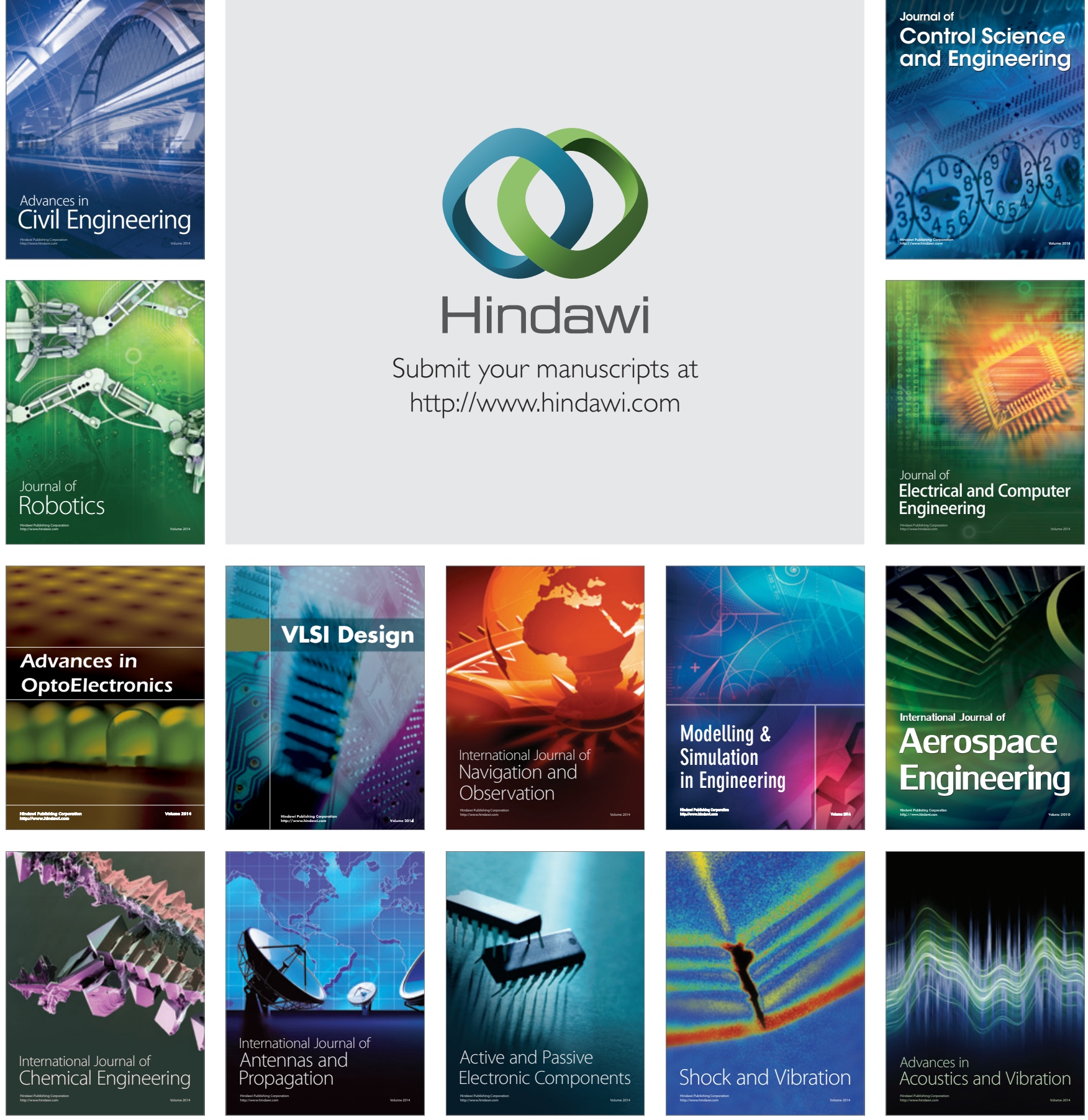